\title{
Pharmakoökonomische Untersuchungen zur Wirtschaftlichkeit eines 5-FU/Salicylsäure-haltigen Präparates in der Therapie vulgärer Warzen
}

\author{
M. Augustin 1 \\ F. Bross ${ }^{4}$ \\ R. Rychlik ${ }^{2}$ \\ I. Zschocke ${ }^{3}$
}

\author{
Medico-Economic Studies on the Economy of SFU/SA Drugs in the Treatment \\ of Vulgar Warts
}

\section{Zusammenfassung}

Hintergrund: 1) Salicylsäure (SA) und 5-Fluorouracil (5-FU) sind effektive Wirkstoffe in der Warzentherapie. 2) In Deutschland werden zunehmend Daten zum Nutzen und zur Wirtschaftlichkeit von Arzneimitteln auf dem Level I der evidenzbasierten Medizin benötigt.

Fragestellung: Wie hoch sind die Wirksamkeit und der klinische Nutzen eines Arzneimittels mit 0,5\% FU und 10\% SA (Verrumal ${ }^{\circledR}$ Lsg.) in der Therapie von a) vulgären und b) plantaren Warzen? Methodik: 1. Ökonomische Modellierung von a) Therapie vulgärer Warzen im Vergleich zu Salicylat-Pflaster und b) Therapie von Plantarwarzen im Vergleich zur Lasertherapie aus der Perspektive der Leistungserstatter. Eingesetzt wurde in beiden Modellierungen jeweils ein zweiarmiges Entscheidungsbaum-Modell (decision tree model) mit den Outcomes „Abheilung“ sowie „Behandlungskosten“ und „Kosten pro Abheilung“.

Ergebnisse: Bei der Behandlung einer vulgären Warze am Finger errechneten sich $€ 29,76$ mittlere Kosten für 5-FU/Salicylsäure und $€ 61,71$ für die Guttaplast-Behandlung. Die Kosten für die Abheilung eines Behandlungsfalles betrugen $€ 42,51$ bei 5-FU/ Salicylsäure-Behandlung und $€ 72,60$ für die Guttaplast-Behandlung. Bei der Behandlung eines Plantarwarzenbeets betrugen die mittleren Kosten der gesamten Therapie $€ 36,19$ für die 5-FU/Salicylsäure-Behandlung und $€ 127,48$ für die Lasertherapie. Die Kosten für die Abheilung eines Behandlungsfalles betrugen dementsprechend $€ 51,70$ für 5-FU/Salicylsäure und $€ 141,64$ für die Lasertherapie. Somit fand sich für 5-FU/Salicylsäure Lsg ein Kos-

\section{Abstract}

Background: 1) Salicylic acid (SA) and 5-Fluorouracil (5-FU) are effective substances in wart therapy. 2) In Germany, increasing data on the benefit and the economic efficiency of drugs at Level I of evidence-based medicine are needed. Questions: How great are the clinical benefits and economy of a medication containing $0.5 \% \mathrm{FU}$ and $10 \% \mathrm{SA}$ (Verrumal ${ }^{\circledR}$ Lsg.) in the therapy of vulgar and plantar warts? Methods: Three-step procedure - 1 . Systematic literature analysis, 2. Metaanalyse of the randomised-controlled studies (RCTs), 3. Economic modelling compared to a) Salicylate patches, b) Laser therapy from the perspective of the reimburser. Results: 1. The efficacy of FU-therapy was tested in a total of 625 patients ( $\mathrm{n}=8$ RCTs) with vulgar warts and 101 patients ( $\mathrm{n}=4$ RCTs) with plantar warts. The therapy effect across all studies in Verrucae vulgares was $68.3 \%$ response (complete healing) for FU vs. $18.3 \%$ for the FU-free controls. In the plantar warts, the response was $63.0 \%$ vs. $16.2 \%$. 2. A metaanalysis of $n=7$ RCTs on vulgar warts ( $\mathrm{n}=325$ patients) showed a mean risk difference of 0.42 (CI $0.34-0.50, \mathrm{p}<0.05$ ), thus significant superiority of 5-FU + SA over SA. A comparable result was also found for plantar warts. 3. In the economic model, there was a cost advantage for 5-FU/Salicylsäure Sol. per healing of $€ 30.09$ on the average in vulgar warts and $€ 89.93$ for plantar warts. Conclusions: The combination of 5-FU and SA is an effective, beneficial and economic therapy in the treatment of vulgar and plantar warts.

Institutsangaben

${ }^{1}$ FG Gesundheitsökonomie und Lebensqualitätsforschung, Klinik für Dermatologie und Venerologie,

Universitätsklinikum Hamburg-Eppendorf

${ }^{2}$ Institut für empirische Gesundheitsökonomie Burscheidt

${ }^{3}$ Institut für Forschungsmanagement und klinische Studien (IFKS) Freiburg

${ }^{4}$ Universitäts-Hautklinik Freiburg

Korrespondenzadresse

Univ.-Prof. Dr. Matthias Augustin · FG Gesundheitsökonomie und Lebensqualitätsforschung · Klinik für Dermatologie und Venerologie · Univ.-Klinikum HH Eppendorf · Martinistraße 52 · 20246 Hamburg ·

E-mail: m.augustin@uke.uni-hamburg.de

Bibliografie

Akt Dermatol 2005; 31: 328-332 @ Georg Thieme Verlag KG Stuttgart · New York

DOI 10.1055/s-2005-861313 · ISSN 0340-2541 
tenvorteil pro Abheilung von durchschnittlich $€ 30,09$ gegenüber Therapie der vulgären Warzen mit Salicylatpflaster und von $€ 89,93$ gegenüber Therapie der Plantarwarzen mit Lasertherapie. Schlussfolgerung: Die Kombination aus 5-FU und SA stellt in der Therapie vulgärer und plantarer Warzen eine effektive, nützliche und wirtschaftliche Therapie dar.

\section{Hintergrund}

Die Therapie vulgärer Warzen mit einer Zubereitung aus 5-Fluouracil und Salicylsäure hat sich in klinischen Studien als effektiv erwiesen [1 -5]. In einer Metaanalyse konnte die klinische Wirksamkeit dieser Kombinationstherapie auf dem Level 1a der evidenzbasierten Medizin belegt werden [6].

Angesichts der heutzutage großen Wichtigkeit pharmakoökonomischer Fragestellungen ist es von zusätzlichem Interesse, über die klinischen Daten hinaus auch Informationen zur Wirtschaftlichkeit entsprechender Dermatika zu erlangen. Für den Bereich der Warzenbehandlung wie auch in den meisten anderen Bereichen der dermatologischen Therapie fehlen bislang Originaldaten, mit denen die Wirtschaftlichkeit von Therapieverfahren differentiell geprüft wird.

Aus diesem Grunde wurde die vorliegende Studie mit der Zielsetzung durchgeführt, anhand einer pharmakoökonomischen Modellierung nach international üblichen Kriterien $[7,8]$ die Wirtschaftlichkeit von 5-FU/Salicylsäure im Vergleich zu typischen Standardtherapien bei vulgären Warzen und bei Plantarwarzen zu prüfen.

Als repräsentative Beispiele für Therapiealternativen zur Behandlung mit 5-FU/Salicylsäure wurden die Lasertherapie zur Behandlung von Plantarwarzen und die Therapie mit Guttaplast zur Behandlung vulgärer Warzen gewählt. Mit diesen beiden Therapiealternativen wurden häufige, in Leitlinien und Therapieempfehlungen stets erwähnte Behandlungsverfahren berücksichtigt, die sowohl den operativen Bereich wie auch den konservativen Behandlungsansatz repräsentieren.

\section{Methoden}

Die Modellanalyse erfolgte mittels der Entscheidungsbaum-Methode („decision tree analysis“) gemäß den üblichen methodischen Vorgaben pharmakoökonomischer Studien [7-9].

Als Effektivitätsparameter bzw. Endpunkt wurde die Abheilung der Warzen („Response“) gewählt und der Nicht-Abheilung („Non-response“) gegenübergestellt.

Die klinischen Daten für die Modellanalyse (Response rate, dropouts, Therapiezeitraum, Nebenwirkungsprofil) wurden aus einer Metaanalyse von $\mathrm{n}=6$ randomisiert-kontrollierten Studien für vulgäre Warzen bzw. $\mathrm{n}=4$ Studien für Plantarwarzen gewonnen [6].
Pharmakoökonomischer Zielparameter waren die direkten Kosten, welche umgerechnet für die Abheilung der Warzen eines Patienten aufgewendet wurden. Die Perspektive war somit die der Kostenträger. Als relevante Komplikationen wurden Superinfektionen der Wunden angesehen und mit eigenen Entscheidungspfaden weitergeführt. Diese sind zwar aufgrund der antimikrobiellen Wirkung der Salicylsäure im Verlaufe der 5-FU/Salicylsäure-Behandlung selten, können jedoch schon bei Therapiebeginn vorliegen und stellen dann kostenrelevante Vorbedingungen dar.

Die ärztlichen Honorare wurden nach EBM errechnet, der Punktwert wurde mit 4 Cent taxiert. Für Arzneimittel und Verbandsmaterial wurden die von den Apotheken mit den gesetzlichen Krankenkassen abgerechneten Kosten zum Stichtag 01. 04. 2003 verwendet. Kostendifferenzen bei den Verbandsmaterialien zwischen verschiedenen Krankenkassen wurden gemittelt.

Ausgehend von den gewählten alternativen Therapieverfahren wurde zunächst für jedes Verfahren von einem Expertenpanel, bestehend aus zwei Dermatologen, einem Ökonomen und einem Statistiker, ein klinisches Szenarium beschrieben, nach dem diese Behandlung typischerweise verläuft. Grundlage hierfür waren sowohl die Angaben zur Anwendung und Dosierung in den Fachinformationen wie auch die Daten aus den bekannten Publikationen. Im Falle der Lasertherapie wurde ferner auf die anonymisierten Behandlungsdaten von 20 zufällig ausgewählten Patienten der Laserambulanz der Universitäts-Hautklinik Freiburg zurückgegriffen.

Zugrunde gelegt wurde die Behandlung

- eines $4 \times 6 \mathrm{~cm}$ großen Plantarwarzenbeetes an der Ferse eines erwachsenen Patienten von $75 \mathrm{~kg} \mathrm{KG}$ und 1,80 m Größe bzw.

- die Behandlung einer erbsgroßen vulgären Warze am Zeigefinger eines erwachsenen Patienten von $75 \mathrm{~kg}$ KG und 1,80 m Größe.

Folgende Annahmen wurden hinsichtlich der klinischen Szenarien getroffen:

Laserbehandlung von Plantarwarzen $(4 \times 6 \mathrm{~cm})$ : Der Behandlungszyklus umfasst im komplikationsfreien Verlauf 4 Sitzungen, bei zusätzlichen Komplikationen (Superinfektion) bis zu zwei weitere Sitzungen. Zusätzliche Kostenfaktoren bei Superinfektionen waren zwei weitere Wiedervorstellungen, ein Abstrich mit Bakteriologie sowie als zusätzliche Medikamente Paracetamol 500- und Panoral 500-Kapseln.

Plantarwarzen mit 5-FU/Salicylsäure: Die Behandlung von Plantarwarzen mit 5-FU/Salicylsäure (Verrumal Lsg.) wurde gemäß dem üblichen klinischen Vorgehen in der Poliklinik der Universitäts-Hautklinik Freiburg vorgenommen. Demgemäß wurden drei Vorstellungstermine in Anrechnung gebracht, bei Superinfektion als Komplikation ein weiterer Termin. Die Arzneimittelkosten wurden spezifisch für die 5-FU/Salicylsäure-Behandlung errechnet, die weiteren Kosten analog zu den bei der Lasertherapie genannten Kosten.

Vulgäre Warzen mit Guttaplast: Hier wurden standardmäßig vier Sitzungen vorgesehen, von denen zwei der Warzenabtragung und zwei der Befundbesprechung dienen. Bei Superinfek- 


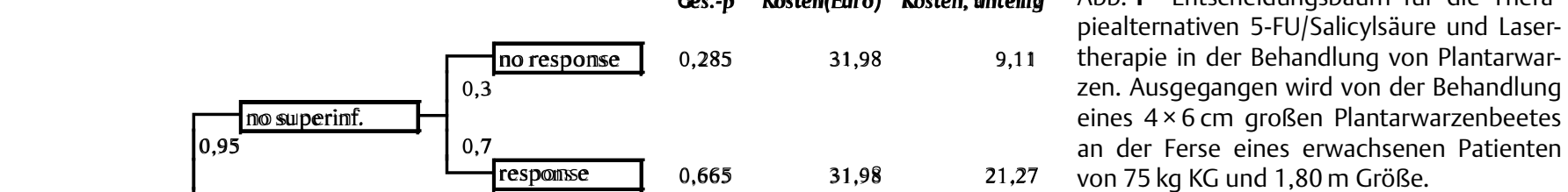
Verrumal

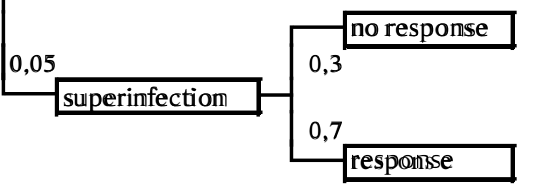

0,015

0,035

Summe

1,00

Kosten/Abheîl.
Abb. 1 Entscheidungsbaum für die Therapiealternativen 5-FU/Salicylsäure und Lasertherapie in der Behandlung von Plantarwareines $4 \times 6 \mathrm{~cm}$ großen Plantarwarzenbeetes an der Ferse eines erwachsenen Patienten von $75 \mathrm{~kg}$ KG und 1,80 m Größe.
Kosten(Euro) Kosten, anteilig

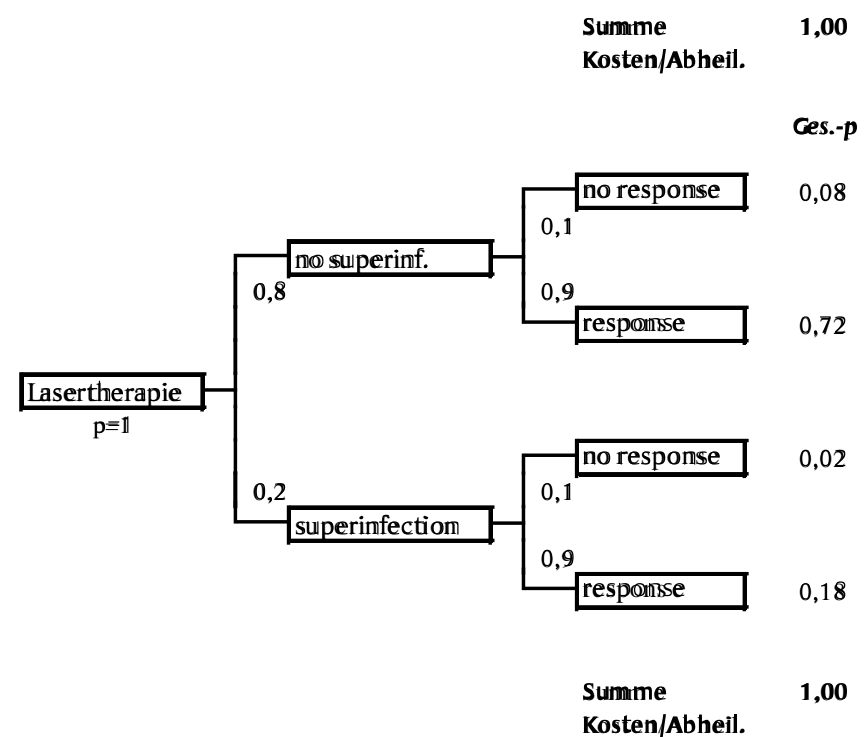

tion kam ein erneuter Besuch hinzu. Die zusätzlichen Kosten für die Superinfektionen errechnen sich wie oben ausgeführt.

Aus diesen Vorgaben wurde ein detaillierter musterhafter Behandlungsablauf erstellt und den einzelnen Therapieschritten jeweils die assoziierten direkten Kosten zugeordnet. Aufgrund der Literaturdaten aus der Metaanalyse bzw. eigens in der Universitäts-Hautklinik Freiburg erhobener Statistiken wurden den verschiedenen Verlaufsformen der Behandlungen (Superinfektion vs. keine Superinfektion; Response vs. Non-response) Eintretenswahrscheinlichkeiten zugeordnet und diese mit den Kosten der jeweiligen Verläufe multipliziert. Errechnet wurden dadurch die mittleren Kosten pro Fall. Nach Division durch die Ansprechrate (response rate) ergaben sich hieraus die Kosten pro abgeheiltem Fall.

Nach Abschluss der primären Analysen wurden univariate, eindimensionale Sensitivitäts-Analysen durchgeführt, um die Stabilität der erzielten Ergebnisse zu prüfen. Folgende Faktoren wurden variiert:

- Anzahl von Einbestellungen (Reduktion um zwei Sitzungen),

- Erhöhung der Komplikationsrate für 5-FU/Salicylsäure um $50 \%$,

- Reduktion der Ansprechrate für 5-FU/Salicylsäure um 10\%,

- Halbierung und Verdopplung des Arzneimittelverbrauchs,

- Erhöhung oder Senkung des Punktwertes der KV um 30\%,
- Änderung der Berechnungsziffern für die ärztlichen Leistungen im gesetzlichen Rahmen.

\section{Ergebnisse}

Bei der Behandlung eines $4 \times 6 \mathrm{~cm}$ großen Plantarwarzenbeets betrugen die mittleren Kosten der gesamten Therapie $€ 36,19$ für die 5-FU/Salicylsäure-Behandlung (Verrumal Lsg.) und $€ 127,48$ für die Lasertherapie. Die Kosten für die Abheilung eines Behandlungsfalles betrugen dementsprechend $€ 51,70$ für 5-FU/ Salicylsäure und $€ 141,64$ für die Lasertherapie (Abb.1).

Bei der Behandlung einer vulgären Warze am Finger errechneten sich $€ 29,76$ mittlere Kosten für 5-FU/Salicylsäure (Verrumal Lsg.) und $€ 61,71$ für die Guttaplast-Behandlung (Abb.2). Die Kosten für die Abheilung eines Behandlungsfalles betrugen $€ 42,51$ bei 5 -FU/Salicylsäure-Behandlung und $€ 72,60$ für die Guttaplast-Behandlung.

Nach Abschluss der primären Analysen wurde eine SensitivitätsAnalyse durchgeführt, um die Stabilität der erzielten Ergebnisse zu prüfen. Hier zeigte sich, dass die Variation der folgenden Parameter keinen Einfluss auf das Ergebnis einer Überlegenheit oder Äquivalenz von 5-FU/Salicylsäure gegenüber Vergleichstherapien aufweist: 


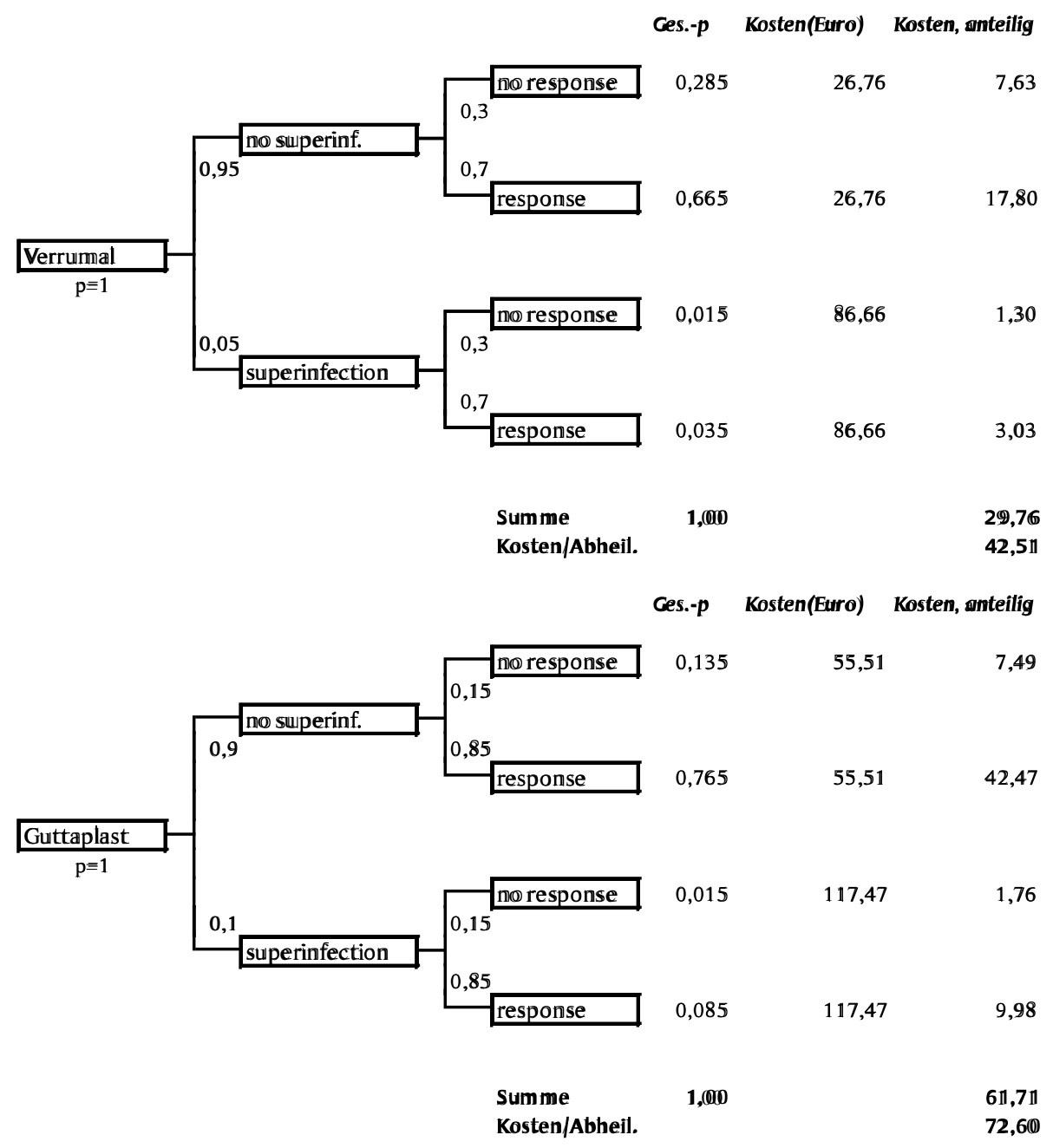

Abb. 2 Entscheidungsbaum für die Therapiealternativen 5-FU/Salicylsäure und Guttaplast in der Behandlung von vulgären Warzen. Ausgegangen wird von der Behandlung einer erbsgroßen Warze am Zeigefinger eines erwachsenen Patienten von $75 \mathrm{~kg} \mathrm{KG}$ und 1,80 $\mathrm{m}$ Größe.

Summe $\quad 1,00$

9,76

42,51

- Variation der Anzahl von Einbestellungen (Reduktion um zwei Sitzungen),

- Erhöhung der Komplikationsrate für 5-FU/Salicylsäure um $50 \%$,

- Reduktion der Ansprechrate für 5-FU/Salicylsäure um 10\%,

- Halbierung und Verdopplung des Arzneimittelverbrauchs,

- Erhöhung oder Senkung des Punktwertes der KV um 30\%,

- Änderung der Berechnungsziffern für die ärztlichen Leistungen im gesetzlichen Rahmen.

In beiden Behandlungsszenarien ist somit die 5-FU/SalicylsäureBehandlung wirtschaftlicher als die analysierten Vergleichstherapien.

\section{Diskussion}

Für die Therapie vulgärer Warzen wurden bislang in der internationalen Literatur keine Wirtschaftlichkeitsdaten publiziert.

Lsg.). Als international übliche Methode wurde eine Entscheidungsbaum-Analyse gewählt, mit der die Kosten-Nutzen-Relation von 5-FU/Salicylsäure im Vergleich zu typischen Vergleichstherapien und Behandlungsszenarien gegenübergestellt werden konnte.

Sowohl im Vergleich zur Lasertherapie bei Plantarwarzen wie auch im Bereich der Therapie vulgärer Warzen fand sich aus pharmakoökonomischer Sicht eine Überlegenheit der 5-FU/Salicylsäure-Lösung gegenüber den Vergleichstherapien. Die Sensitivitätsanalyse unterstützt die Hypothese, dass diese Lösung gegenüber anderen Therapieformen wie Laserbehandlung oder Salicylatpflaster-Behandlung einen Benefit sowohl hinsichtlich einer Verbesserung des klinischen Scores wie auch der Lebensqualität und nicht zuletzt der Kostenfaktoren darstellt.

Die vorliegenden Daten stellen eine praxisnahe Modellierung dar, deren Aussagekraft jedoch zukünftig durch eine prospektive Studie gestützt werden sollte.

Ziel der vorliegenden Studie war die Prüfung der Wirtschaftlichkeit der Therapie von vulgären und plantaren Warzen mit einem Kombinationspräparat aus 5-FU und Salicylsäure (Verrumal 
Literatur

${ }^{1}$ Breitbart EW. Controlled clinical studies of a new wart medication based on fluorouracil. Z Hautkr 1979; 54 (19): 853-855

${ }^{2}$ Katsarou-Katsari A, Papageorgiou M, Stratigos I. Klinische Studie mit 5-Fluorouracil und Salicylsäure bei der Behandlung von Warzen. Notabene medici 1986; 4: 229-230

${ }^{3}$ Meyer-Rohn J, Schmersahl P. External administration of fluorouracil in the management of warts. Z Hautkr 1978; 53 (19): 697-700

${ }^{4}$ Schmidt H, Jacobsen FK. Double-blind randomized clinical study on treatment of warts with a fluorouracil-containing topical preparation. Z Hautkr 1981; 56 (1): $41-43$
${ }^{5}$ Wolff K. Ergebnis einer klinischen Untersuchung von Verrumal ${ }^{\circledR}$ gegen Placebo, doppelblind und randomisiert im Halbseitenversuch. Hermal Internal report - Gutachten 1980; 1 -3

${ }^{6}$ Zschocke I, Schlöbe A, Cummerow R, Hartmann A, Augustin M. Wirksamkeit und Nutzen eines 5-FU/Salicylsäure-haltigen Präparates in der Therapie vulgärer und plantarer Warzen - systematische Literaturübersicht und Metaanalyse. JDDG 2004; 2: 187-193

${ }^{7}$ Rychlik R (Hrsg). Gesundheitsökonomie. Enke Verlag, Stuttgart: 1999

${ }^{8}$ Hannoveraner Konsensus-Gruppe. Deutsche Empfehlungen zur gesundheitsökonomischen Evaluation - Revidierte Fassung des Hannoveraner Konsens. Gesundheitsökonomie und Qualitätsmanagement 1994; 4: A62 - A65

${ }^{9}$ Rychlik R. Venen

\section{Buchbesprechung}

\section{Dermatosen aus drei Kontinenten. Bildatlas der vergleichenden Dermatologie}

Schmeller W., Bendick C., Stingl P.

Stuttgart: Schattauer, 2005. 229 S. m. 328 Farbfotos.

Geb. 129€, ISBN 3-7945-2339-3

Der Farbbildatlas der vergleichenden Dermatologie mit über 100 verschiedenen, alphabetisch angeordneten Krankheitsbildern von A wie Acne bis $Z$ wie Zoster, die von den drei Autoren unter schwierigen Bedingungen aus drei Kontinenten (Europa, Asien, Afrika) zusammengetragen worden sind, ist der beste Beweis dafür, dass die Völker näher zusammengerückt sind. Anders formuliert, dass wir in EINER Welt leben. Europa ist ein Zuwanderungsland. Dieser Tatsache ist es zuzuschreiben, dass wir Dermatologen auch mit Hautkrankheiten auf farbiger (gelber und schwarzer) Haut vornehmlich in Ballungsgebieten konfrontiert werden. Dank dieses speziellen Atlas erhalten wir die Möglichkeit nachzuschauen, um welches Krankheitsbild es sich handeln könnte, da uns stets der Vergleich zu den uns auf weißer Haut geläufigen Krankheiten mitgegeben ist. Das Verdienst dieses Buches besteht außerdem darin, dass wir wie durch ein Schlüsselloch einen teilweise erschreckenden Einblick in die katastrophalen Verhältnisse in den Ländern der Dritten Welt und den sehr beschränkten therapeutischen Maßnahmen gewährt bekommen. Bedrückend sind - exemplarisch genannt - Bilder von einem Kind mit Tinea profunda capitis oder von einem Erwachsenen, der mit Lepraplaques geradezu übersät ist. Solche Bilder sind zum Hin- und nicht zum Wegschauen gedacht. Sie lösen Betroffenheit aus und haben den Kollegen Bendick veranlasst, wieder in Phnom Penh tätig zu werden.

Die einzelnen Krankheitsbilder werden auf Doppelseiten nach einem einheitlichen Schema besprochen. Therapeutische Maßnahmen werden eher gestreift, was für dieses Werk auch ausreichend ist. Die alphabetische Abfolge lässt ein schnelles Auffinden der Krankheiten auch ohne das vorhandene Sachverzeichnis zu. Eine einseitige Bibliografie mit den wichtigsten weiterführenden Büchern rundet das Werk ab. Das Buch gibt uns Ärzten zudem die Möglichkeit, unsere Betroffenheit durch eine Spende an zwei Vereine, „Ärzte helfen hautkranken Kindern in Afrika e.V.“ und „Society for Dermatology in the Tropics e.V." auszudrücken. Einige Anmerkungen ergänzender und kritischer Art seien gestattet: Benzoylperoxid zählt nicht mehr zu den Standardtherapeutika einer Akne. Die infantile Akropustulose kommt bei Kindern jeder Hautfarbe vor, auf schwarzer am häufigsten.

Nicht die Pityriasis alba, sondern die Pityriasis versicolor wird auch als Kleieflechte bezeichnet. Diese kommt bei Diabetes mellitus oder AIDS nicht öfter vor. Die Behandlung der Tinea capitis im Kindesalter mit Terbinafin bzw. Itraconazol ist und wird in Deutschland nicht zugelassen. Griseofulvin bleibt das Mittel der Wahl. Kritisch zu sehen ist die keratolytische Behandlung mit salizylsäurehaltigen Externa, vor allem in der Therapie des Milchschorfs oder einer Psoriasis capillitii bei Kindern. Es sind Hörstürze bei Erwachsenen nach der berühmten (vielleicht eher berüchtigten) salizylsäurehaltigen Ölkappe beschrieben, da Acidum salicylicum leicht von der Haut aufgenommen wird. Dank unserer in manchen Dingen nicht nachzuvollziehenden Gesundheitsreform ist Salizylsäure rezeptierbar, hingegen Urea pura, die zur (unschädlichen) Keratolyse getreu der Hippokratischen Maxime des „nil nocere“ einsetzbar ist, nicht.

Fazit: Ein aufrüttelnder und instruktiver Atlas, der seinen stolzen Preis wert ist und allen niedergelassenen und in der Klinik tätigen Dermatologen ans Herz gelegt werden darf. Der deutsche Dichterfürst Johann Wolfgang von Goethe hat es auf den Punkt gebracht: „Was man weiß, sieht man erst“ (Schriften zur Kunst, Propyläen 1798-1800).

C. Oster-Schmidt, Altenkirchen 